\title{
Pictorial essay: Artifacts in cardiac CT: A practical system of understanding and correction
}

\author{
Hemant B. Telkar \\ Department of Radiology, Jupiter Heart Scan, Mumbai, India
}

Correspondence: Dr. Hemant B Telkar, Jupiter Heart Scan 756 Mancherji Joshi Marg, Parsi Colony Garden View Building, Mumbai, India. E-mail: telkar@vsnl.com

Artifacts are defined as various patterns that appear in images, but are not present in the original object. There are three types of artifacts encountered during CT coronary angiography; patient related, procedure related and reconstruction related.

\section{Patient-Related}

These may be due to
A. Obesity
B. Breath-hold
C. Movement
D. Dense calcium or metal

\section{A. Obesity \\ In patients weighing more than $80 \mathrm{~kg}$ or with large breasts, there is increased noise resulting in wall irregularity and difficulty in differentiating from plaques [Figures 1, 2].}

To eliminate noise, we can:

a. Try to scan the patient at a lower pitch, with overlapping images.

b. Use a smoother kernel.

c. Use a higher mAs [Figure 2].

d. Use more contrast.

\section{B. Breath-Hold}

Holding one's breath for even as short as 8 to 10 seconds can be a problem for some patients [Figure 3]. To eliminate this problem, the patient must practice breath-holding before the procedure and if required an attendant can be placed in the room next to the patient's head for nose-holding. Slice collimations of $1.2 \mathrm{~mm}$ can help reduce the scan time, while still maintaining adequate image quality [Figure 4].

Despite all these steps, in about $1-5 \%$ of patients, breathing related artifacts still occur.

\section{Patient Movement}

Good communication with the patient before the procedure eliminates anxiety and fear and reduces motion related artifacts.

\section{Streak Artifacts}

The presence of metal, especially after a bypass-surgery or dense calcium can lead to streak artifacts [Figure 5].

\section{Procedure-Related}
A. ECG
B. Poor planning
C. Contrast-related.

\section{A. ECG-Related}

1. Metallic electrodes on the anterior chest wall.

2. Improper placement of electrodes, resulting in baseline tremors, waveform artifacts and shallow trace.

1. Metallic electrodes on the anterior chest wall

Due to immense improvement in the resolution of the scanners, streak artifacts due to metal in the ECG electrodes are not much of a problem on the 64-slice scanners [Figure 6].

\section{Improper placement of electrodes}

Poor contact of the leads or electrodes with the skin or physical contact of the female breasts with the electrode wires may result in an abnormal ECG trace.
a. Abnormal amplitudes [Figure 7]
b. Baseline tremors [Figure 8]
c. Wave-form artifact [Figure 9]

The above changes can be prevented by proper placement of electrodes, with good use of jelly for proper contact and to affix the leads after raising the patients' hands above their heads, so as to prevent creasing of the skin under the lead pads. Occasionally, we can change the position of the leads, such as, by putting them on the back [Figure 10]. Breasts can be strapped if they come in the way. 

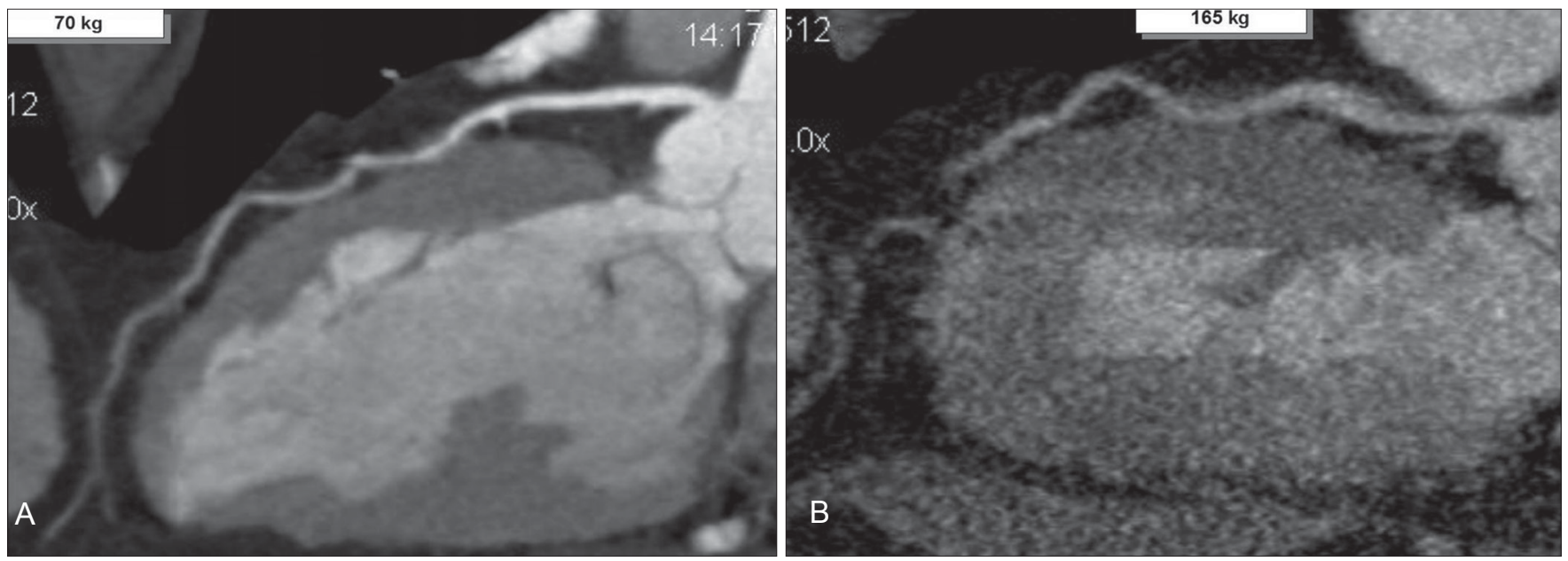

Figure 1 (A-B): $70 \mathrm{~kg}$ individual (A) shows a high signal-to-noise as against a grainy image due to low signal-to-noise in a $165 \mathrm{~kg}$ individual (B).
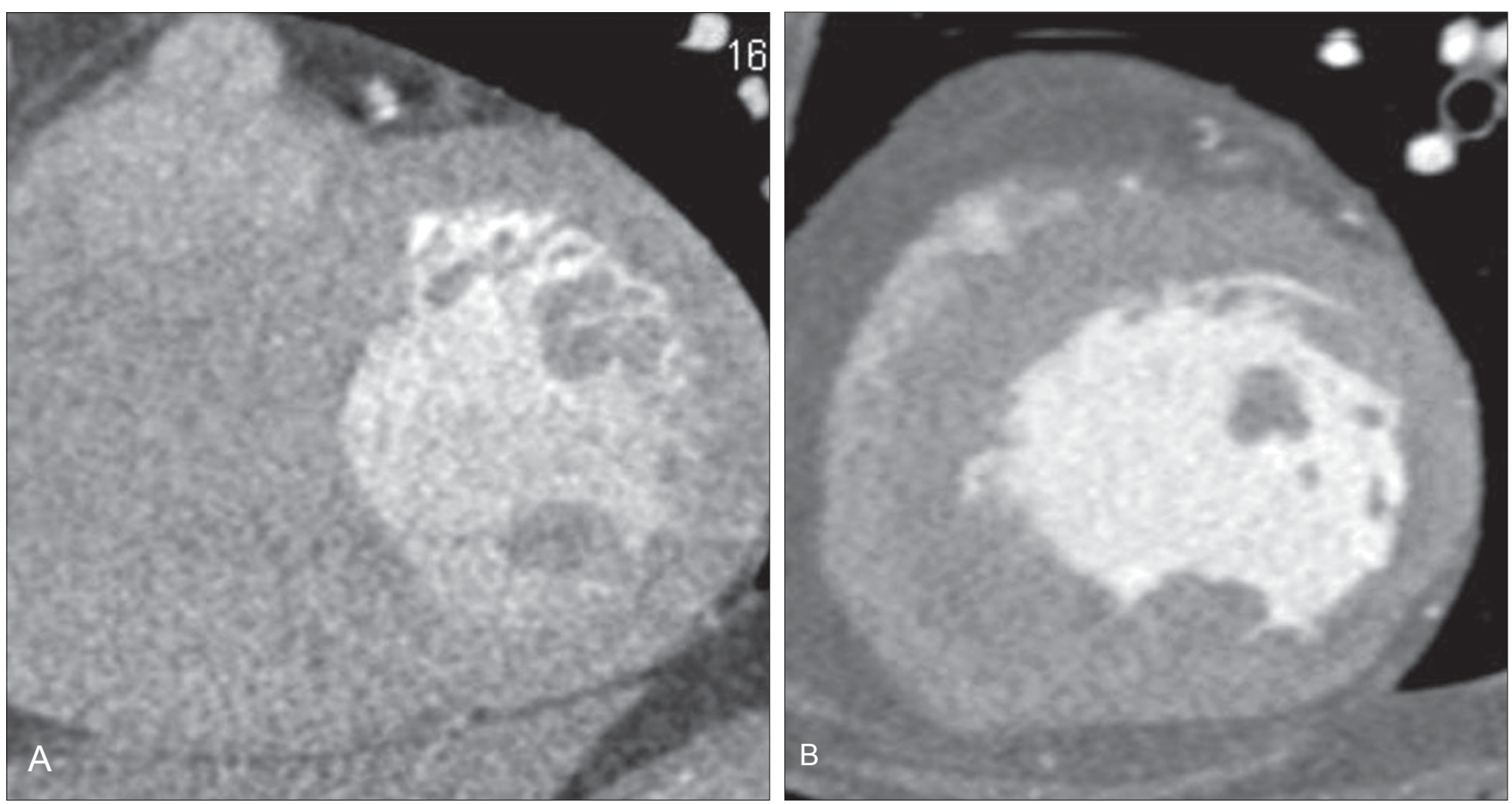

Figure 2 (A-B): Short axis images show a grainy image at $650 \mathrm{mAs}(\mathrm{A})$ and a much smoother image at $850 \mathrm{mAs}(\mathrm{B})$. This however means increased radiation.

\section{B. Poor Planning}

Poor planning can generate unwanted artifacts and pseudostenosis [Figure 11]. To prevent this from happening, we should scan from the level of the inferior border of the arch of the aorta downwards. The calcium score axial data can be used to identify the left main (LM). We should then start higher than this level [Figure 12].

\section{Contrast-Related}

1. Streak Artifact

Streak artifacts may occur due to contrast in the SVC, at the time of starting the scan. The SVC at this time is still filling with undiluted contrast from the veins and this may give rise to dense streak artifacts over the RCA
[Figure 13]. To avoid this, we can inject from the left arm and use a saline flush.

Contrast in the right ventricle (RV) [Figure 14A] also causes an artifact over the RCA, which can be eliminated by using a saline chaser [Figure 14B]. However in patients with a poor cardiac output, the RV opacification may still be dense and cause artifacts.

2. Slab Artifacts

These are caused by the improper use of contrast medium. Often, the concentration of contrast medium is locally too high or the contrast medium injection may have started too early. This gives rise to "contrast" slabs [Figure 15]. 


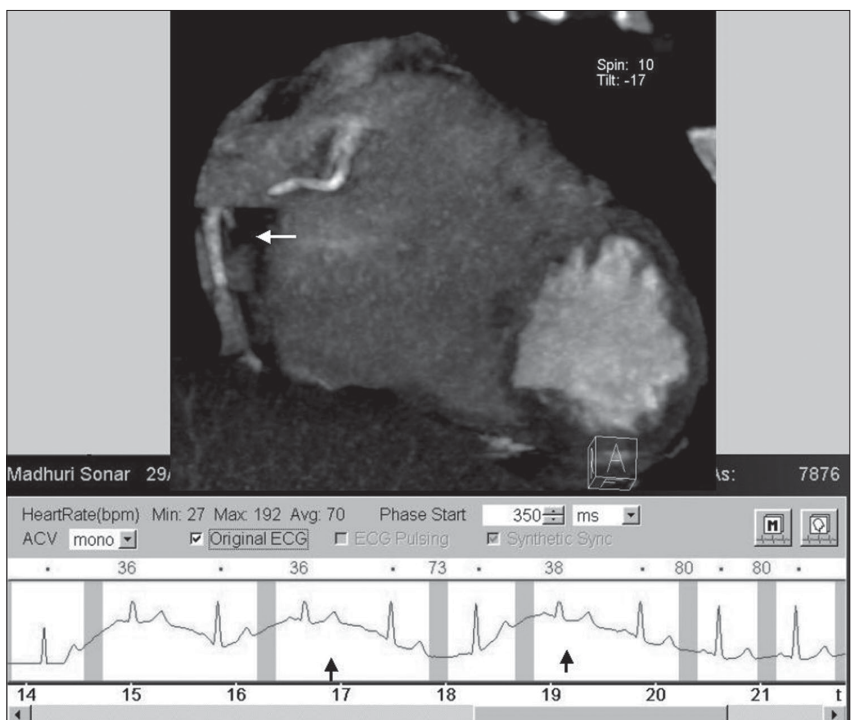

Figure 3: Free breathing during a study is seen as undulation in the baseline ECG resulting in an artifact (white arrow) along the RCA.

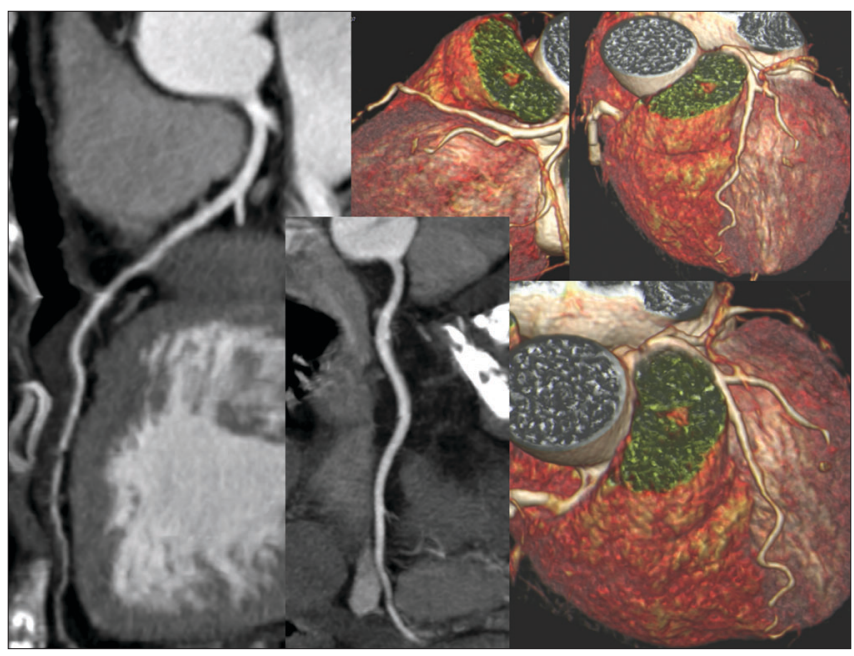

Figure 4: A good quality cardiac CT study done with $1.2 \mathrm{~mm}$ collimation, in a scan time of $6-8$ seconds.
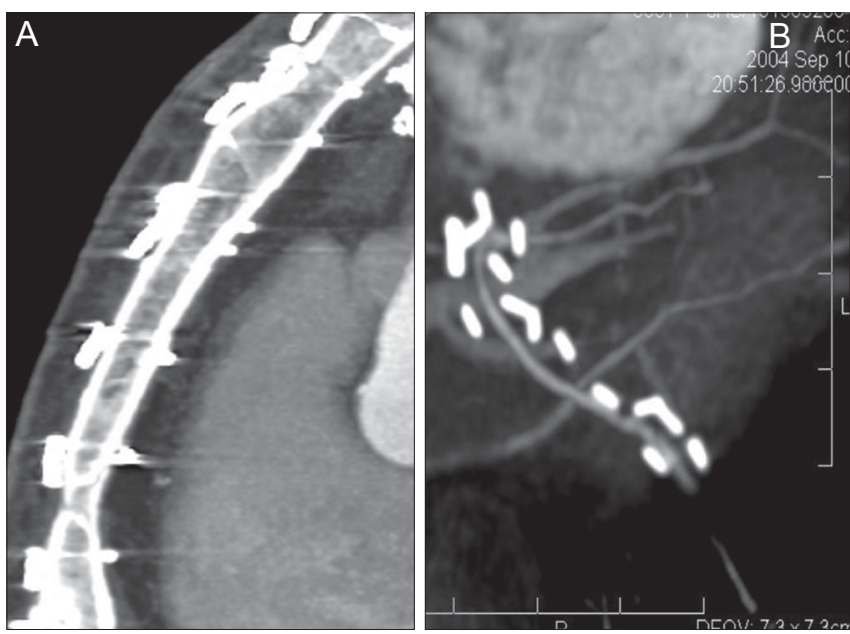

Figure 5: MIP images show streak artifacts from metal sutures $(A)$, whereas those from the surgical clips (B) are minimal, in a patient who is post-bypass.

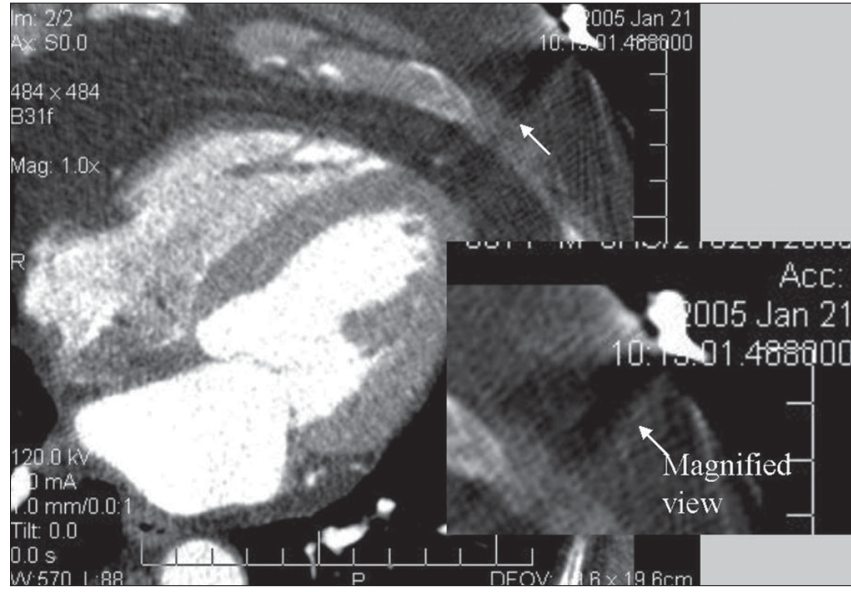

Figure 6: Axial image of a cardiac CT dataset shows streak artifacts (white arrow) from the metal in the ECG lead.

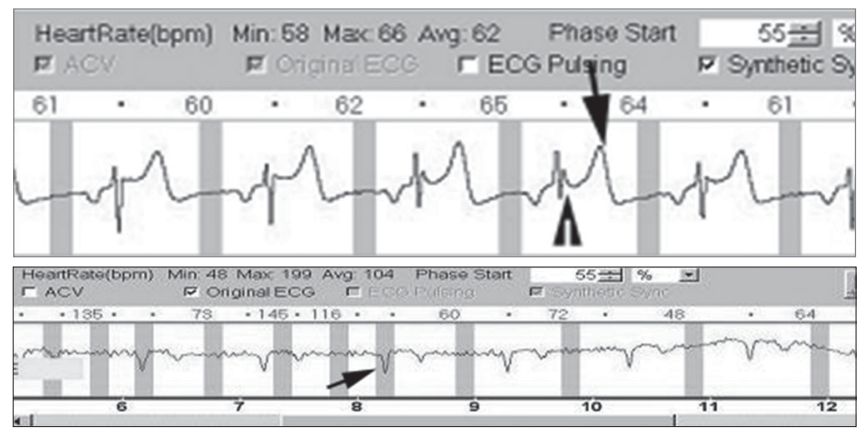

Figure 7: Abnormal amplitudes. The ECG trace (A) shows a T wave (arrow) of larger amplitude than the R wave (arrowhead). Another trace (B) shows very low amplitude $\mathrm{R}$ waves (arrow).

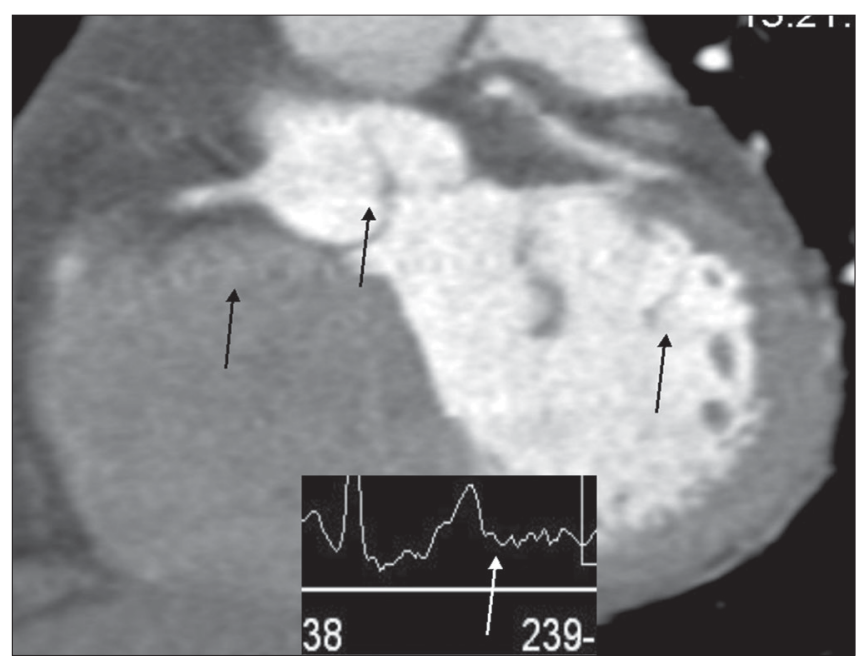

Figure 8: Baseline tremors. The reconstruction image shows fuzzy lines (arrows) in the image due to a poor baseline ECG trace (white arrow, see inset)

\section{Reconstruction-Related}

These include artifacts due to:

A. High heart rate

B. Variable heart rate 


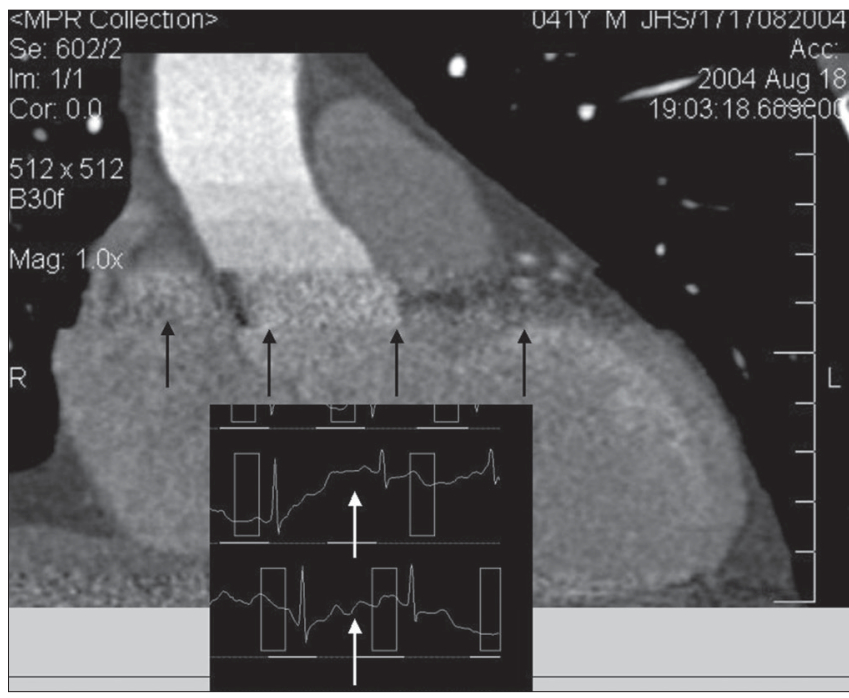

Figure 9: Waveform artifact. The poor ECG trace (white arrows in inset) is responsible for the step-artifacts (black arrows).

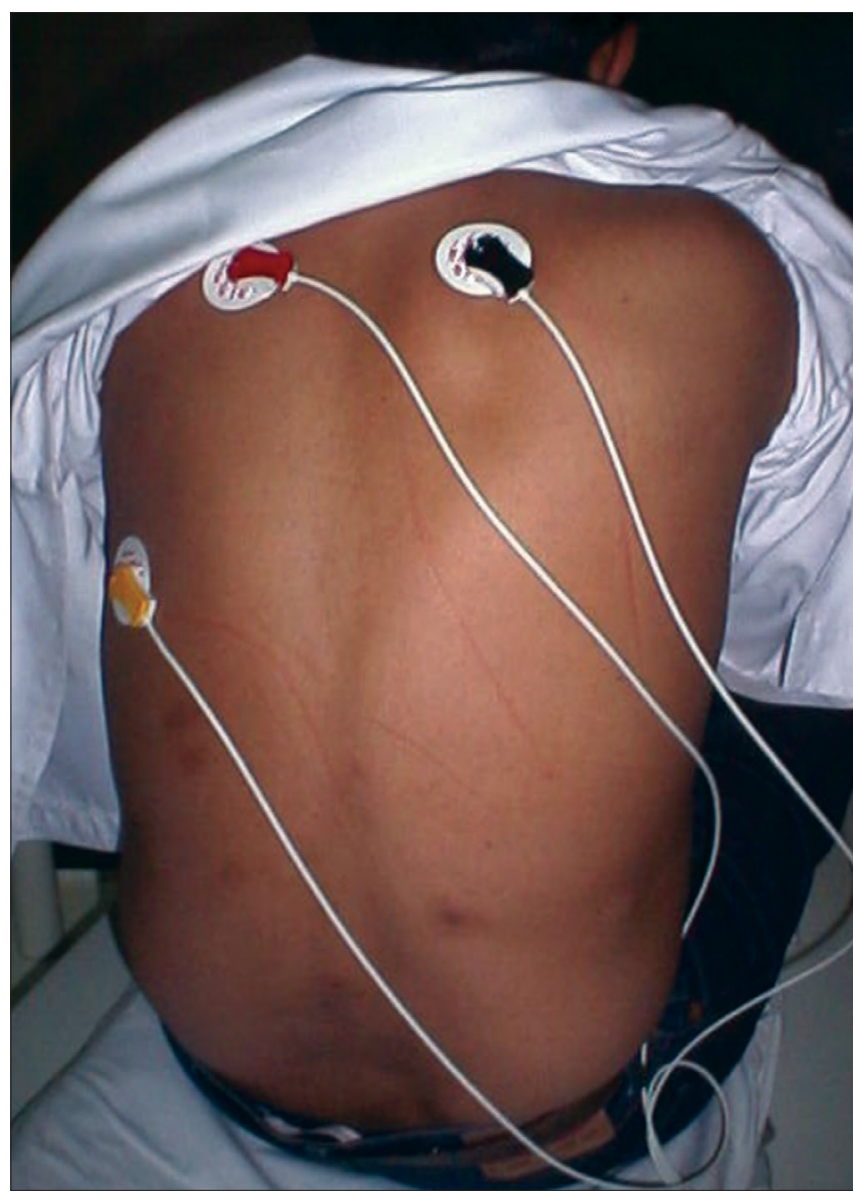

Figure 10: Alternate ECG lead placement. The photograph shows ECG leads placed on the back.
C. Selection of incorrect phase
D. Step artifacts due to misregistration

\section{A. High Heart Rate}

The RCA exhibits the highest velocity movement during the
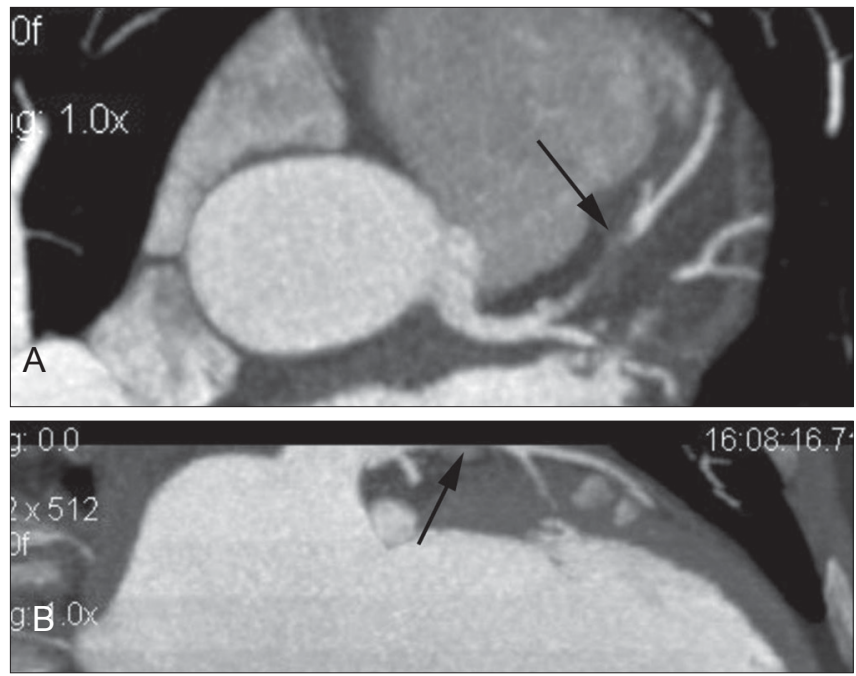

Figure 11 (A-B): Improper placement of the acquisition band leads to incomplete visualization of the LAD (arrow) in the axial (A) and coronal (B) planes.

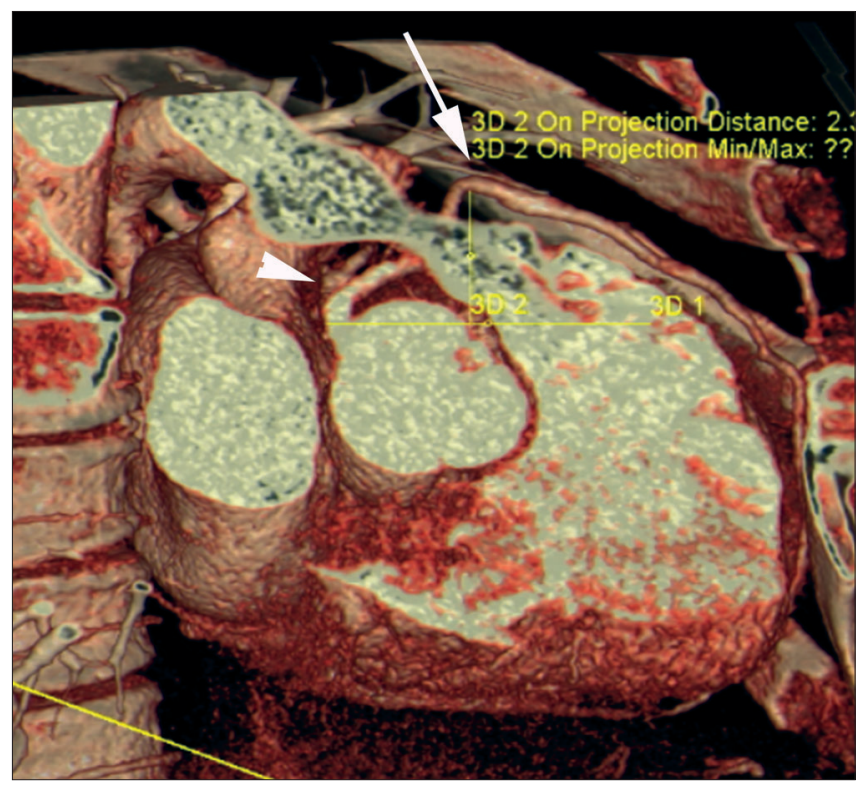

Figure 12: Volume rendered image shows that the LAD (arrow) is positioned $2.3 \mathrm{cms}$ above the axial plane of the LM (arrowhead) origin.

cardiac cycle [Figure 16] and the greatest positional change in the $\mathrm{x}$ and $\mathrm{y}$ planes, followed by the circumflex branch of the left coronary artery, the left main coronary artery and the left anterior descending artery.

The heart rate should be as low as possible, so as to broaden the mid-diastolic band to get better temporal resolution in the data acquisition band. This is the reason for giving beta-blockers.

\section{B. Variable Heart Rate}

The problem of a variable heart rate was more pronounced in 4-slice and 16-slice scanners, where the feeling of warmth after contrast injection, would lead to an increase in the heart rate in the latter part of the acquisition. 


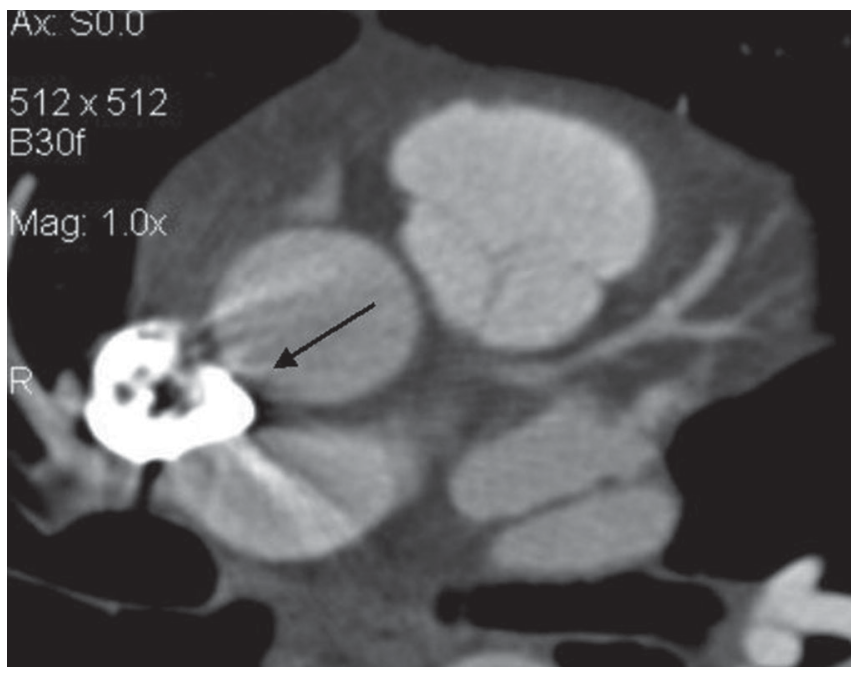

Figure 13: Axial image shows dense streak artifacts from the SVC (arrow).

Measures to eliminate artifacts in the above conditions i) Multi-sector reconstructions.

In dual-sector reconstruction, the temporal window can be reduced to a maximum of $1 / 4^{\text {th }}$ of the gantry rotation time [Figure 17]. This increases the flexibility and placement of the temporal window in the shortened R-R interval at high heart rates.

ii) Manipulation of the ECG trace

Various tools are available for manipulation of the ECG trace, which include one or more of the following capabilities.

a. Drag the syncs forward/backward

b. Insert extra syncs

c. Disable and delete syncs

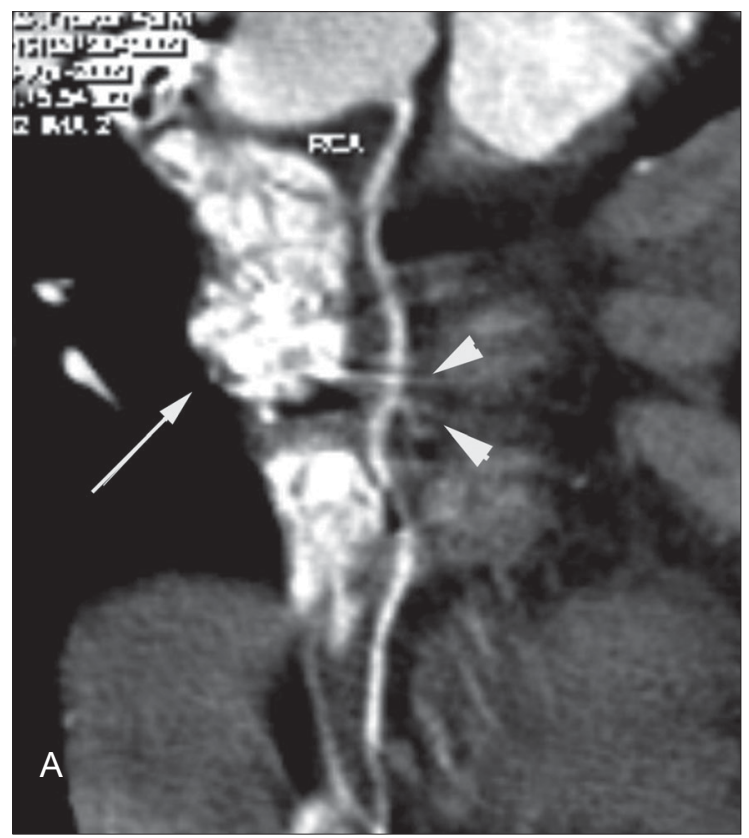

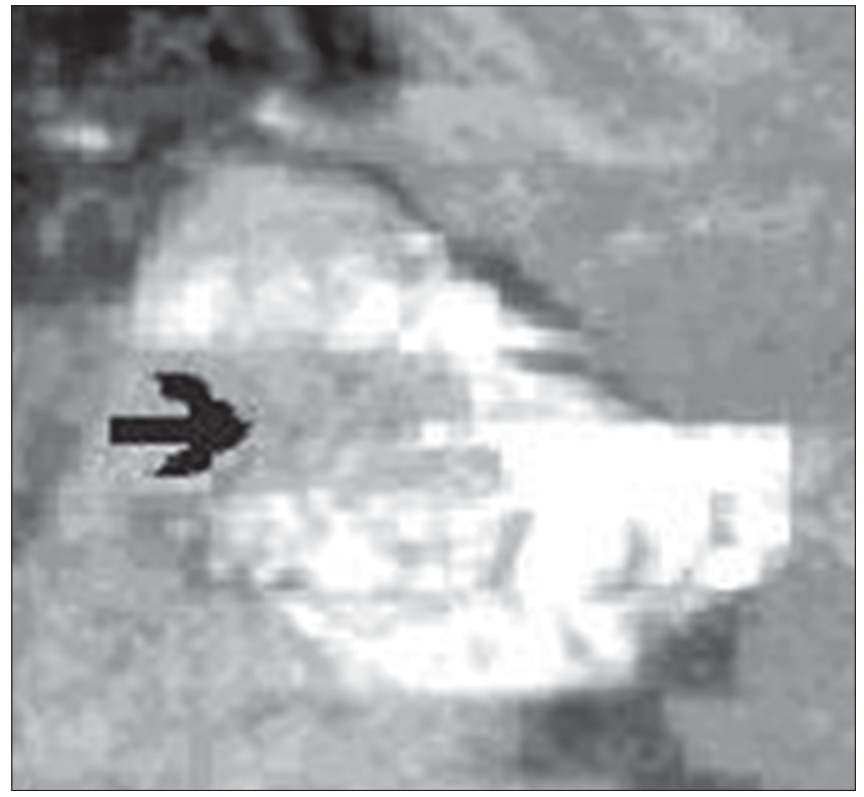

Figure 15: MIP image show a "slab" artifact (arrow) due to differential contrast opacification.

a. Drag the syncs forward /backward

Step artifacts due to improper placement of syncs can be eliminated or reduced by changing the position of these syncs [Figure 18].

b. Insert extra sync

In cases where the difference between two adjacent heart beats is high, inserting an additional sync helps in averaging the ECG data and resultant uniformity in the trace pattern [Figure 19].

c. Disable and delete syncs

Figure 14 A-B: Contrast in RV (arrow) causes steak artifacts over the RCA (arrowhead), which are eliminated after the use of a saline flush (B), which results in markedly reduced contrast concentration in the RV (arrow). Note the RCA (arrowhead). 

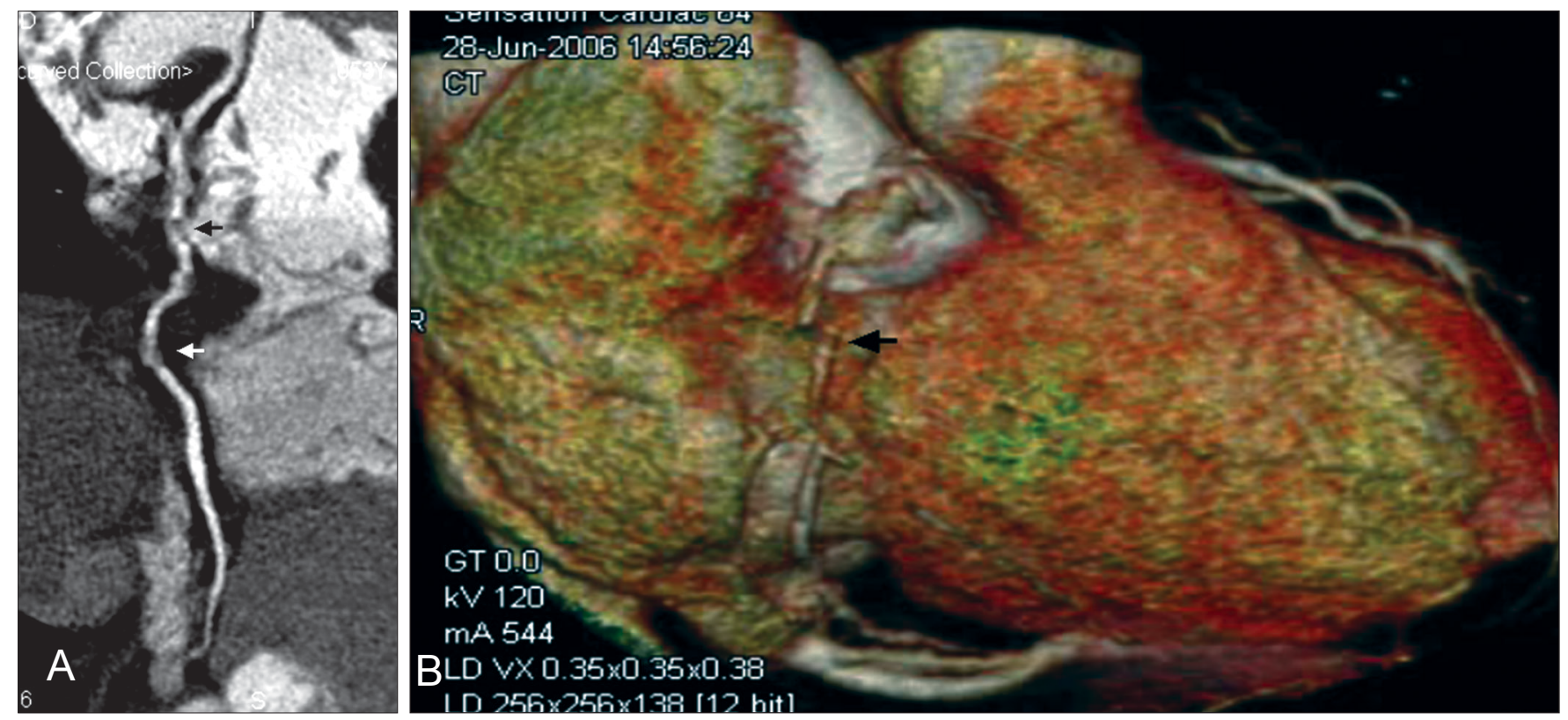

Figure 16 (A-B): MPR image $(A)$ and volume rendered image $(B)$ show step artifacts (arrows) due to RCA motion.

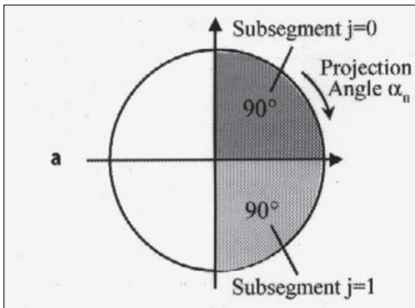

Parallel Projection Range

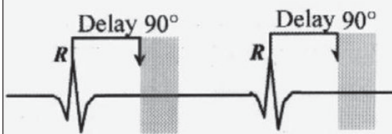

Time t, Projection Angle $\alpha_{\mathrm{n}} \longrightarrow$

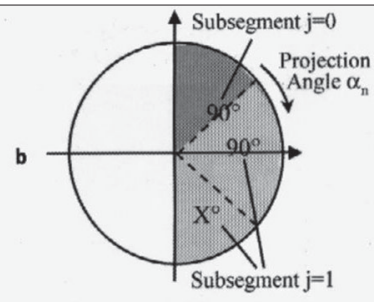

Parallel Projection Range

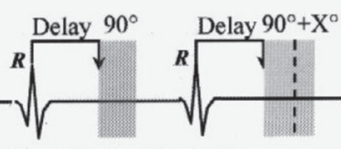

Time t, Projection Angle $\alpha_{\mathrm{n}} \longrightarrow$

Figure 17: Diagram showing the concept of multi-sector reconstruction, using the ACV (Adaptive Cardio Volume TM) mode for Siemens scanners.

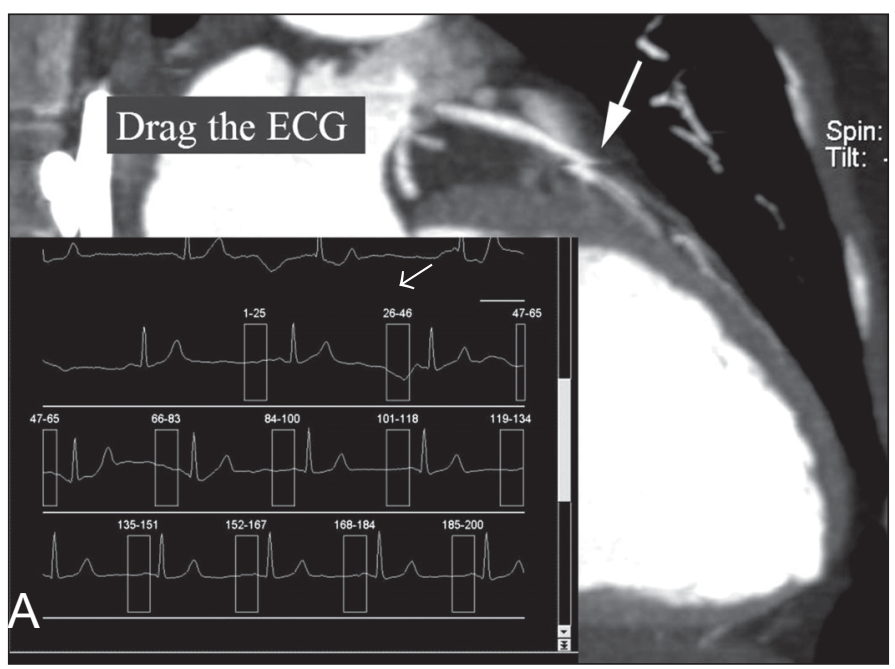

To overcome ectopic beats, the corresponding syncs can be disabled [Figure 20]. In this situation, the ectopic beat is not read during image reconstruction and the data from the previous heartbeat is used to fill in the image; this negates any image variation in the ECG pattern thereby removing the step in the final image. The disadvantage is that this leads to interpolation across the R-R interval thereby resulting in loss of information and blurring in the final reconstructed image.

\section{Incorrect Phase}

The choice of an incorrect phase can give rise to artifacts [Figure 21]. Choosing the correct phase eliminates these artifacts [Figure 22].

The optimum phases are as follows

Figure 18 (A-B): The precorrection image (A) shows an artifact in the LAD (arrow) due to the acquisition band falling in late diastole/early systole. After dragging the ECG trace manually so that it lies in the middiastolic band the LAD artifact in the postcorrection image (B) is eliminated (arrow). 


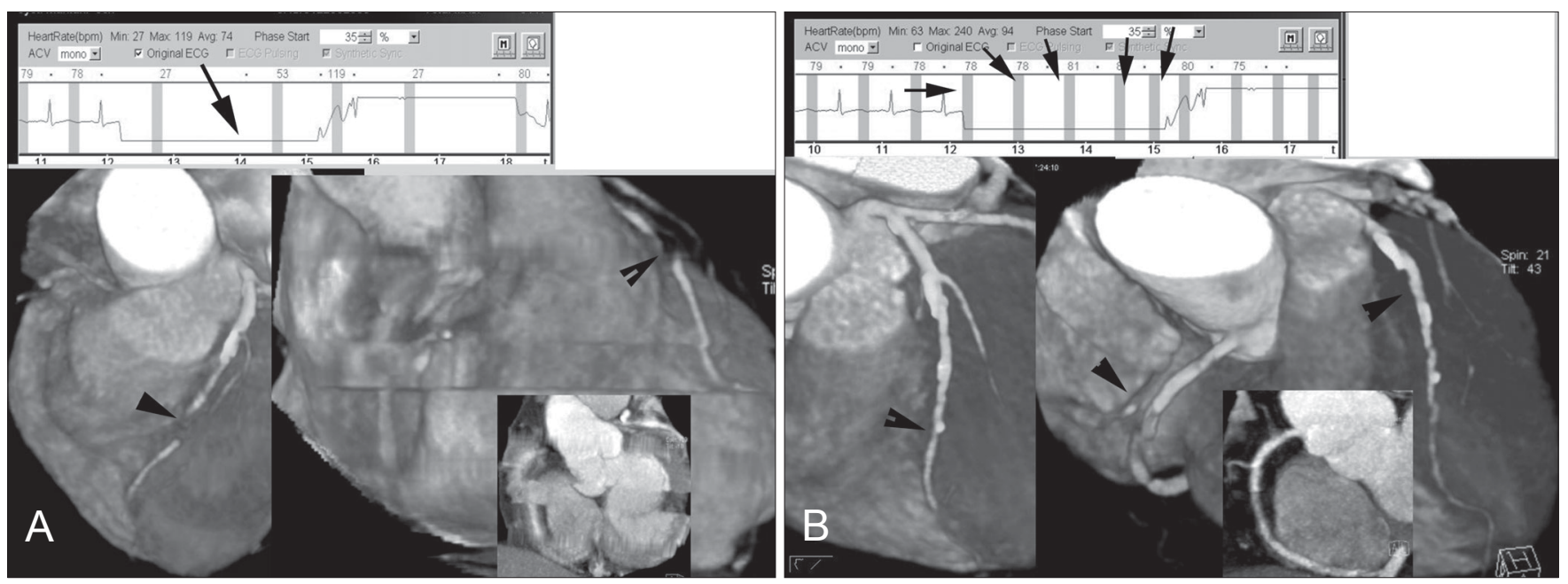

Figure 19 (A-B): The pre-correction image (A) shows a breathing artifact leading to a flat line (arrow) in the ECG trace and LAD artifacts (arrowheads). By inserting multiple syncs (arrows), the post-correction image (B) shows elimination of the LAD artifacts (arrowhead).
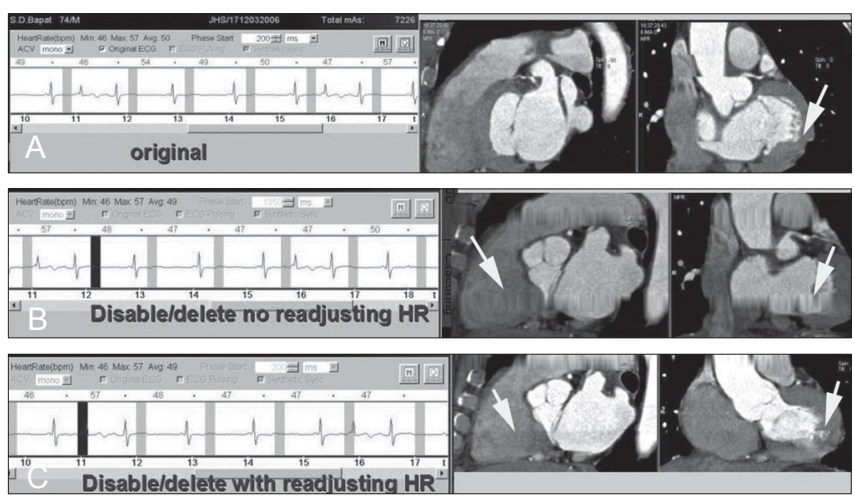

Figure 20 (A-C): The original image (A) shows a bad step artifact (arrow) across the LV cavity. Disabling the offending sync (B) without heart-rate adjustment leads to a blurring artifact (arrow), whereas both artifacts are eliminated (arrows) after readjusting the heart rate as well (C).

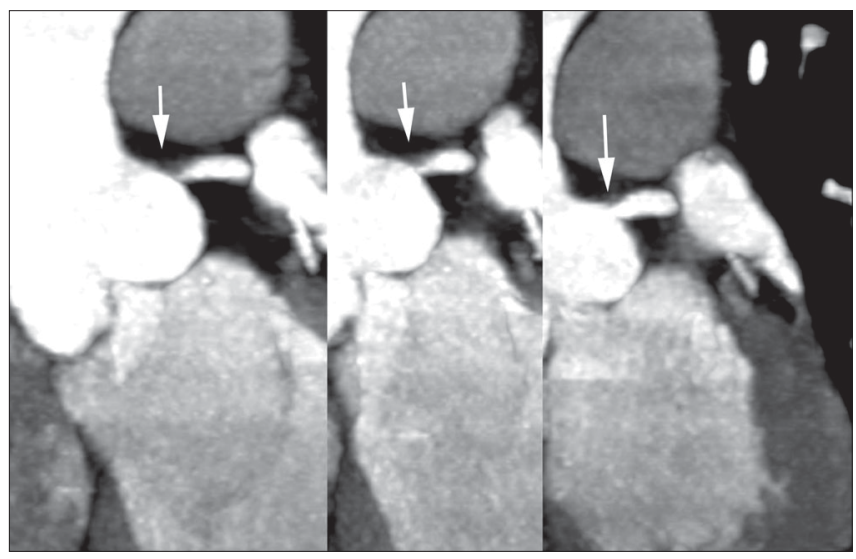

Figure 21: The LM shows different appearances in different phases, simulating narrowing (arrows).

$<60 \mathrm{bpm}-70 \%$ and 850/900 zms

61- $65 \mathrm{bpm}-70 \%$ for the left side and 30\% for the RCA

$>65$ bpm - 250/300ms (high HR)

However assessing at least two data sets, one using the
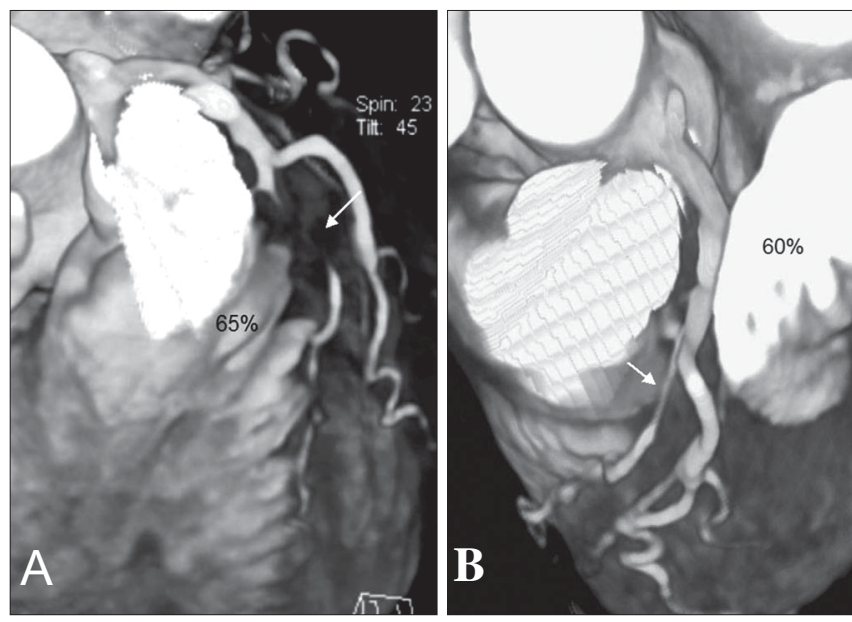

Figure 22 (A-B): A poor choice of phase shows an artifact (arrow) in the $L A D$ at $65 \%$ reconstruction $(A)$, which disappears (arrow) at $60 \%$ reconstruction $(B)$.

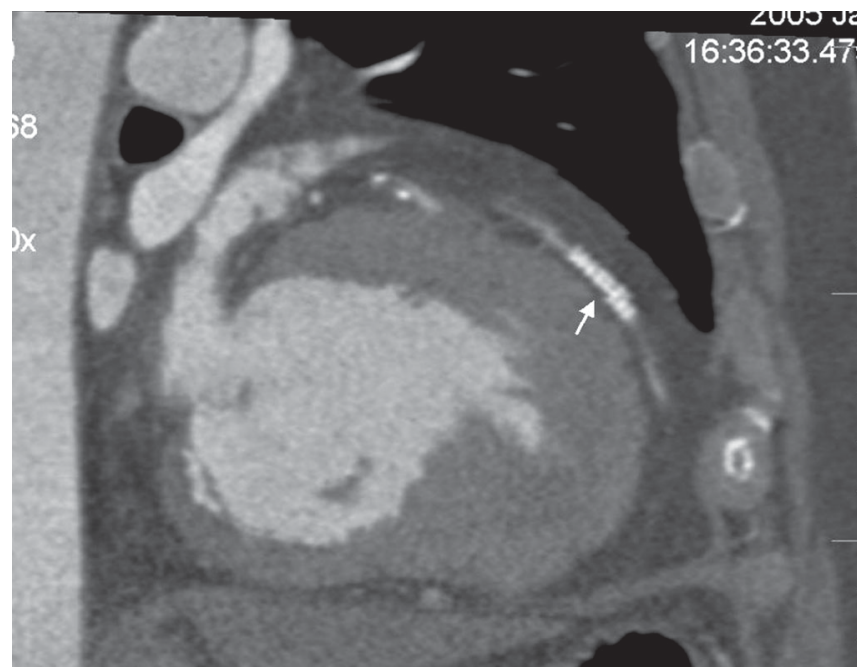

Figure 23: MIP image shows a mis-registration step artifact involving an LAD stent (arrow). 


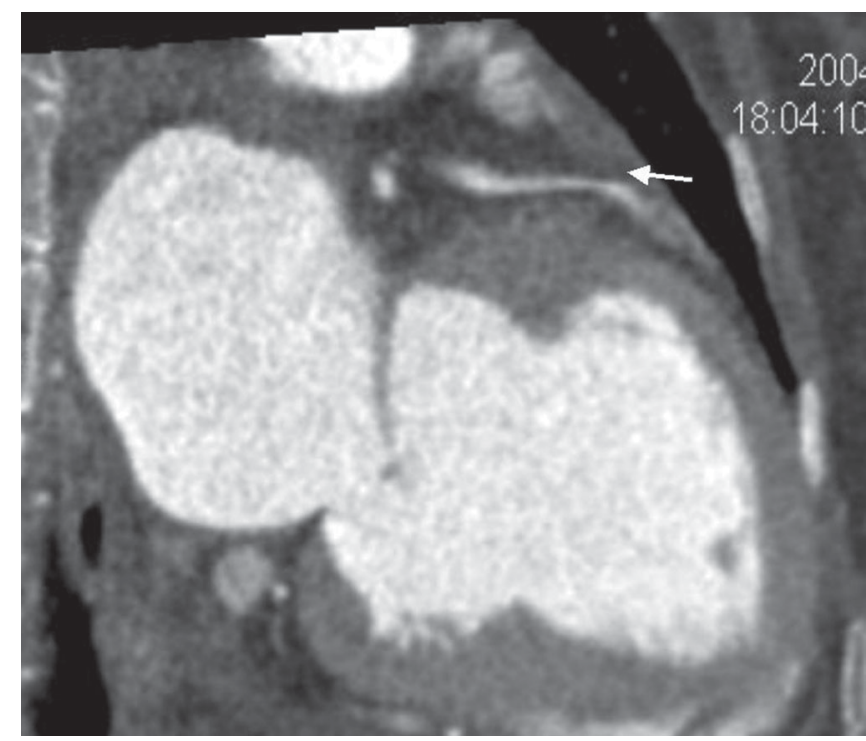

Figure 24: MIP image shows a mis-registration step artifact involving the proximal LAD (arrow).

percentage of the R-R interval and the other using the ms time before or after the preceding $\mathrm{R}$ wave, helps in avoiding artifacts and in falsely interpreting stenotic lesions.

D. Step Artifacts due to Mis-registration
Step artifacts due to intrinsic mis-registration of data are known [Figures 23, 24]. These can be eliminated by using one or more of the following measures:

i. Try more than one reconstruction phase

ii. Try to edit the ECG trace

iii. Sometimes a thicker reconstruction can be used, e.g. 1 $\mathrm{mm}$ instead of $0.75 \mathrm{~mm}$

iv. A multi-sectorial reconstruction may be used.

\section{Conclusion}

Artifacts that result from technical errors in image data acquisition and interpretation may be avoided with appropriate planning and execution of the scanning procedure, including instruction and practice of the patient in breath-holding, as well as the optimal selection of anatomic coverage, scanning delay, pitch and reconstruction window. To improve interpretation errors, it is essential to use multiple (at least two) reconstructions tailored to each case to avoid mis-interpretation and bad image quality.

This article is based entirely on the author's experience and highlights practical issues.

Source of Support: Nil, Conflict of Interest: None declared.

\section{Advertisement}

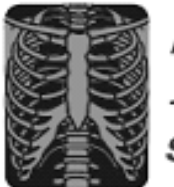

\section{Teleradiou $\log y$}

India's pioneering Teleradiology Company, Teleradiology Solutions is recruiting radiologists for its state of the art reading center in Whitefield, Bangalore. The center also incorporates a polyclinic and diagnostic imaging center with a fully equipped imaging department. Research and teaching opportunities are provided and there is a strong focus on academics. We provide an excellent career opportunity to for young and dynamic radiologists.

Interested persons may E-mail sunita.maheshwari@telradsol.com or call Ms. Tina Victor at $40187500 / 06$.

Contact Info: Teleradiology Solutions Pvt. Ltd., Plot No. 7G, Council Khata 180/63

Vishveshwaraiah Industrial Area

Whitefield, Bangalore - 560048, India.

Ph: 40187500/ 40187506

Fax: 41103411 